\title{
Time dilation induced by object motion is based on spatiotopic but not retinotopic positions
}

\author{
Ricky K. C. Au ${ }^{1,2 *}$, Fuminori Ono ${ }^{1}$ and Katsumi Watanabe ${ }^{1,3}$ \\ ${ }_{1}^{1}$ Research Center for Advanced Science and Technology, The University of Tokyo, Tokyo, Japan \\ 2 Japan Society for the Promotion of Science, Tokyo, Japan \\ ${ }^{3}$ Japan Science and Technology Agency, Saitama, Japan
}

\section{Edited by:}

Hirokazu Tanaka, Salk Institute, USA

Reviewed by:

Hirokazu Tanaka, Salk Institute, USA

Makoto Miyazaki, Yamaguchi

University, Japan

*Correspondence:

Ricky K. C. Au, Research Center for Advanced Science and Technology,

The University of Tokyo, 4-6-1

Komaba, Meguro-ku, Tokyo 153-8904,

Japan.

e-mail: ricky@fennel.rcast.u-tokyo.

ac.jp
Time perception of visual events depends on the visual attributes of the scene. Previous studies reported that motion of object can induce an illusion of lengthened time. In the present study, we asked the question whether such time dilation effect depends on the actual physical motion of the object (spatiotopic coordinate), or its relative motion with respect to the retina (retinotopic coordinate). Observers were presented with a moving stimulus and a static reference stimulus in separate intervals, and judged which interval they perceived as having a longer duration, under conditions with eye fixation (Experiment 1) and with eye movement at same velocity as the moving stimulus (Experiment 2). The data indicated that the perceived duration was longer under object motion, and depended on the actual movement of the object rather than relative retinal motion. These results are in support with the notion that the brain possesses a spatiotopic representation regarding the real world positions of objects in which the perception of time is associated with.

Keywords: motion, retinal position, spatiotopic, time perception

\section{INTRODUCTION}

The existence of all entities in the physical world is defined by three dimensions in space and one dimension in time. One special thing about time, which is quite different from that of space, is that we do not possess a sensory modality or sensory receptors which can directly sense time. Time does not have mass, nor emits physical energy that our sensory machineries can directly sense with. We can directly feel the size of a physical object by vision, touch, and other senses; but we require the perception of changes from these sensations, which signals the onset and offset of an event, in order to perceive time.

Although the history of studying time perception is relatively short in comparison to other areas in perception such as vision, previous studies have discovered a range of interesting properties in relation to human perception of the length of time about visual events. When asked to judge the relative durations of two visual events, human observers reported differential perception of duration subject to the influence of various physical properties (Fraisse, 1984) such as visibility (Terao et al., 2008), intensity (Matthews et al., 2011), and complexity (Roelofs and Zeeman, 1951; Schiffman and Bobko, 1974), as well as subjective size (Ono and Kawahara, 2007) of the stimulus being viewed. Reducing visibility can apparently compress the perceived time interval (Terao et al., 2008), which has also been demonstrated during visual saccades (Morrone et al., 2005). Regarding stimulus intensity, weak stimuli on a strong intensity background were judged as having longer duration, and such effect appeared to be stronger at longer durations (Matthews et al., 2011). In addition, by manipulating the magnitudes of some non-temporal dimensions including number, size, luminance, and the numeric value of digits, Xuan et al. (2007) found that larger, brighter, and more numerous visual stimuli could create an illusion of lengthened duration. The size of stimuli has also been shown to affect the perceived time of an empty interval (Ono and Kitazawa, 2009).

Change signals time, and motion involves change. We experience motion of ourselves and external objects all the time. Previous psychophysical findings have pointed to the idea that the processing of timing for events with stationary and moving stimuli is dissociated (Aschersleben and Müsseler, 1999). Motion has been found to "dilate" the subjective perception of duration (Brown, 1931, 1995; Verstraten et al., 2005). In addition, higher moving speed and longer moving distance can both lengthen the time perceived (Rachlin, 1966; Brown, 1995). Furthermore, change in motion speed can also alter the perceived duration. Using temporal judgment and reproduction tasks, Matthews (2011) reported that objects moving with constant speed appeared to last longer than decelerating stimuli, which in turn seemed to last longer than accelerating ones in temporal judgments, although the difference between decelerating and accelerating motion disappeared in reproduction tasks. In addition to object moving across the frontal plane of the observer, using stimuli of different sizes to represent approaching or receding objects, Ono and Kitazawa (2010) reported the observation that receding objects appeared to have subjectively longer duration than approaching ones, suggesting that time dilation effect also occurs for motion in depth. While a number of studies had suggested motion as the key factor in inducing time dilation, Kanai et al. (2006) demonstrated that time dilation can in fact be simply induced by flickering stimuli. Comparing to static display, flickering displays produced a time dilation illusion. Kanai et al. (2006) concluded that, rather than the motion, temporal frequency was a more important factor in determining the perceived duration, 
which has its basis at the early stages of processing. However, this idea remained controversial, since later findings showed that speed seemed to be the determinant factor rather than temporal frequency in the time dilation illusion. Through systematic variation of temporal frequency, spatial frequency, and speed of visual stimuli, Kaneko and Murakami (2009) found that speed, rather than temporal or spatial frequency, of the stimulus best described the perceived duration, and the illusion was not due to inaccurate judgment for onset and offset time of moving versus static stimuli. They suggested that the processing of the time dilation illusion is mediated at a stage of higher level of processing.

In the topic of time perception for visual events in motion, one outstanding question remains to be addressed is whether the occurrence of the time dilation effect is based directly on the processing of signals projected onto the retina of the eyes (i.e., a retinotopic representation), or based on a representation created in the brain which locates the real world positions of objects in the environment (i.e., a spatiotopic representation). The latter case might imply that the visual signals have gone to a stage at some higher level of processing by the time when the dilation illusion is established. Previously, it has been shown that visual adaptation to fast moving stimuli could alter time perception in the adapted region (Johnston et al., 2006). Using the method of adaptation with drafting gratings and displacing the fixation to another position after adaptation, Burr et al. (2007) found that, comparing among retinotopic, spatiotopic, and a control position, the perceived duration of test stimulus presented at the spatiotopic position of adaptation was the longest. On the other hand, the perceived stimulus duration at the retinotopic position of adaptation only differed a little from the control condition. Their results suggested that time perception of short visual events are likely to be processed by neural mechanisms with spatially circumscribed receptive fields localized in real world coordinates, but not retinal ones. But is this also the case for brief visual events with object motion?

In the present study, we performed two experiments to answer this question by comparing the perceived duration of a static reference stimulus with a moving stimulus, under conditions with or without eye movement (which leads to near absence or presence of object movement relative to the retina). Experiment 1 aimed to replicate the time dilation effect of object motion with eye fixation. Observers compared the perceived duration of a reference with the comparison stimulus (static or moving) presented for varying time intervals. In Experiment 2, observers kept their eyes fixated on a moving fixation cross, while the comparison stimulus remained physically stationary or moving at the same velocity as the fixation cross. The first condition represented a condition with retinal motion but not physical motion (retinotopic motion), while the second condition with physical motion but not retinal motion (spatiotopic motion). We hypothesized that, if time perception is processed based on the brain's representation of objects in real world coordinates instead of retinal ones, the perceived duration in the spatiotopic motion condition would be longer than that in the retinotopic motion condition.

\section{EXPERIMENT 1: TIME DILATION UNDER OBJECT MOTION WITHOUT EYE MOVEMENT MATERIALS AND METHODS \\ Observers}

Eleven paid volunteers who were all naïve as to the purpose of the study were recruited at the University of Tokyo and participated in the experiment. One of the authors (RA) also participated. Informed consent was obtained prior to the experiment. All observers had normal or corrected-to-normal vision (aged 21-28; eight female).

\section{Stimuli}

Stimuli were programmed in MATLAB using the Psychophysics Toolbox extensions (Brainard, 1997; Pelli, 1997), and were viewed on a CRT monitor at the refresh rate of $100 \mathrm{~Hz}$, with resolution at $800 \times 600$ pixels, controlled by a personal computer running on the Windows XP operating system. Observers viewed the stimuli at a distance of $60 \mathrm{~cm}$ in a quiet and dimly illuminated room. All stimuli appeared white in color $\left(49.02 \mathrm{~cd} / \mathrm{m}^{2}\right)$ against a black background $\left(0.016 \mathrm{~cd} / \mathrm{m}^{2}\right)$ on the screen. The fixation stimulus was a cross $\left(\right.$ size $=0.453^{\circ} \times 0.453^{\circ}$, width $\left.=0.0907^{\circ}\right)$ appeared at the center of the screen. The target was a circular stimulus with diameter of $1.360^{\circ}$.

\section{Experimental design and procedures}

In each trial of the experiment, a reference stimulus and a comparison stimulus were presented on the screen at separate time intervals (Figure 1). Each trial began with presentation of the fixation stimulus which remained at the center of the screen throughout the trial. Observers were instructed to keep focusing on the fixation cross throughout the experiment. The observer triggered the stimulus presentation by pressing the Space bar on the keyboard. After $1000 \mathrm{~ms}$, the reference stimulus (the target circle) appeared at $4.533^{\circ}$ below the fixation point for a constant duration of $1000 \mathrm{~ms}$. After the reference stimulus has disappeared from the screen for $1000 \mathrm{~ms}$, the comparison stimulus was then presented for a random duration of 800,1000 , or $1200 \mathrm{~ms}$. In the Static condition, the comparison stimulus was the target circle appearing at $4.533^{\circ}$ above the fixation point without movement. In the Moving condition, the target circle also always appeared at the same vertical distance above the fixation point, but moved at a constant speed of $11.334^{\circ} / \mathrm{s}$ from left to the right of the fixation point horizontally. We introduced random variation of $-0.136^{\circ}, 0^{\circ}$, or $+0.136^{\circ}$ for the starting and ending positions of the moving stimulus, so that the observer would not be accustomed to an identical stimulus for the same Duration condition. As a result, the starting positions were from $4.533 \pm 0.136^{\circ}, 5.667 \pm 0.136^{\circ}$, and $6.800^{\circ} \pm 0.136^{\circ}$ to the left of the fixation, to the same distance to the right of the fixation, for 800,1000 , and $1200 \mathrm{~ms}$ Duration conditions respectively. Once the presentation of the comparison stimulus ended, the observer judged whether the reference or comparison stimulus had appeared for a longer duration by pressing the Left (for reference stimulus) or Right (for comparison stimulus) key on the keyboard. The experiment was comprised of 2 Motion conditions $\times 3$ Duration conditions $\times 3$ Starting-Ending positions presented in pseudorandom order with 6 repetitions, resulting 


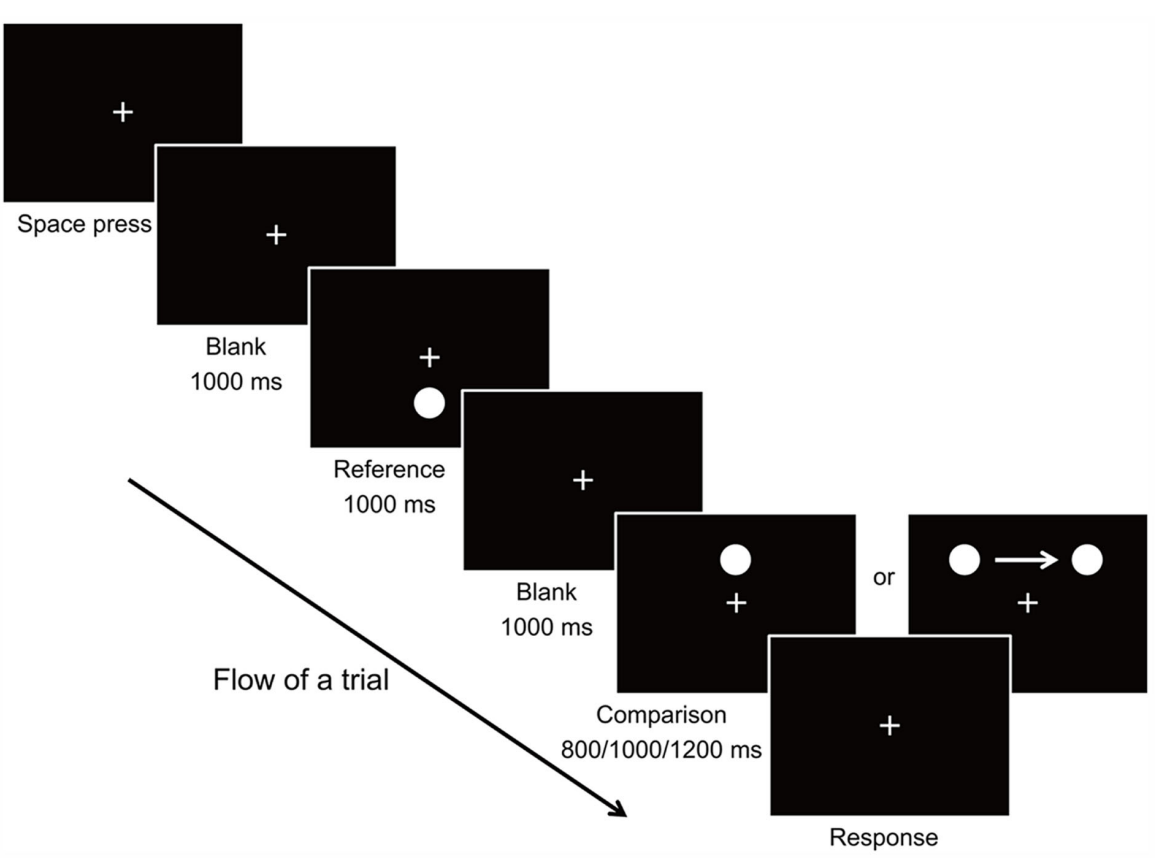

FIGURE 1 | A schematic diagram depicting the flow of a trial in Experiment 1 (main experiment).

in a total of 108 trials. The experiment took about $10-15$ min to complete.

\section{Control experiment}

In the experiment, the comparison stimulus was either moving or remained stationary, while the reference stimulus was always stationary. To examine the possibility that the effect might be due to a novelty effect attributed to the moving comparison stimulus, a control experiment (with the same number of trials) was conducted with the identical setting, with the only difference that the reference stimulus ( $1000 \mathrm{~ms}$ ) always moved across the screen from $5.667^{\circ}$ to the left of the fixation, to the same distance to the right of the fixation. Each observer performed either the main or the control experiment first at counter-balanced order. A break of at least 5 min was given between the performance of the main and control experiment.

\section{RESULTS AND DISCUSSION}

The proportion of trials in which the duration of the target stimulus was perceived to be longer than that of the reference stimulus was computed for the Static and Moving condition, averaged across the 12 observers, and plotted against the 3 durations of presentation (Figure 2).

A $2 \times 3$ repeated measures ANOVA with Motion (Static/Moving) and Duration (800/1000/1200 ms) as the withinsubject factors was performed on the data for the main experiment (stationary reference) to examine effect of object motion on perceived time duration (Figure 2A). The results revealed significant main effects of Motion $[F(1,11)=15.939, p=0.002]$ and Duration $[F(2,22)=214.866, p<0.001]$. A significant Motion $\times$ Duration interaction was also found $[F(2,22)=6.149$, $p=0.008]$. Post hoc paired-sample $t$-tests revealed that the

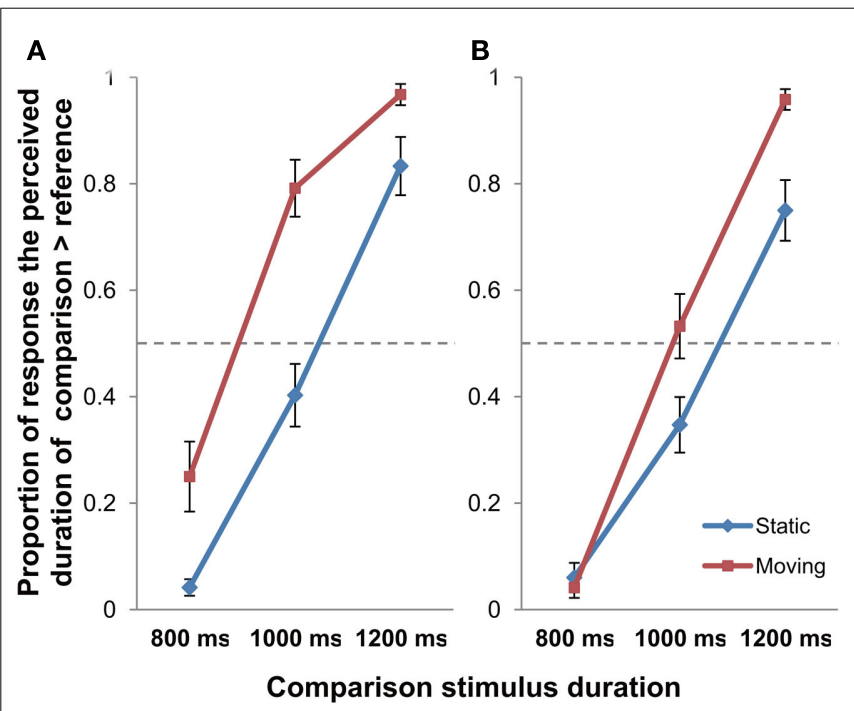

FIGURE 2 |The proportion of response that the comparison stimulus was perceived as longer than the reference stimulus in Experiment 1 [(A) stationary reference; (B) moving reference control experiment]; error bars represent SEM.

difference between Static and Moving conditions were significant at the presentation duration of $800 \mathrm{~ms}[t(11)=-3.264, p=0.023]$ and $1000 \mathrm{~ms}[t(11)=-4.072, p=0.006]$, but it did not reach the significant level for $1200 \mathrm{~ms}[t(11)=-2.245, p=0.139$; all Bonferroni-corrected].

The same $2 \times 3$ repeated measures ANOVA (Motion $\times$ Duration) was also performed on the data of the moving reference control 
experiment (Figure 2B). Results indicated significant main effects of Motion $[F(1,11)=11.174, p=0.007]$ and Duration $[F(2$, $22)=175.846, p<0.001]$. The Motion $\times$ Duration interaction was also found to be significant $[F(2,22)=8.645, p=0.002]$. Post hoc paired-sample $t$-tests reported significant difference between Static and Moving conditions at the presentation duration of $1200 \mathrm{~ms}$ [ $t(11)=-4.586, p=0.002]$, though it failed to reach significance under $800 \mathrm{~ms}[t(11)=0.561, p>0.999]$, and with marginally significant difference under $1000 \mathrm{~ms}[t(11)=-2.717$, $p=0.060]$ presentation duration (all Bonferroni-corrected). As observed in Figure 2, both the main and the control experiment exhibited the same trend of longer time perception under object motion. The control experiment has thus demonstrated that the observed time dilation effect obtained in the main experiment was motion-based, rather than due to novelty effect of stimuli.

To summarize, the data indicated that the time perceived in the condition with the moving object was lengthened in comparison to that without object movement, which was consistent with the results obtained in previous studies in general.

\section{EXPERIMENT 2: TIME DILATION UNDER OBJECT MOTION WITH EYE MOVEMENT}

In Experiment 2, we closely examined whether the effect of time dilation associated with object movement depends on the positional shift of the object image projected on the retina (retinotopic position), or on the localization of actual physical movement of the object as represented in the brain (spatiotopic motion). In Experiment 2, observers were instructed to trace the fixation moving at a constant speed across the screen. As a consequence, a physically static target stimulus would be projected on the retina as moving at the same speed in an opposite direction, whereas a target stimulus moving at the same speed and direction with the fixation cross would create a relatively static image on the observer's retina. If the brain computes the duration of visual events based on the retinal image, the former condition would result in a longer perceived duration. On the other hand, if the brain determines time duration based on a representation with awareness of whether the object is physically moving or not, we would expect the time dilation effect to be shown in the latter condition. With consideration for possible time dilation effect confounded with voluntary smooth pursuit eye movements (Wenke and Haggard, 2009; Schütz and Morrone, 2010), the moving fixation was also employed in the reference stimulus in Experiment 2.

\section{MATERIALS AND METHODS \\ Observers}

Eleven new, paid observers who were naïve as to the purpose of the study, and one of the authors (RA), participated in the experiment (aged 20-28, two female). All observers had normal or corrected-to-normal vision. Informed consent was obtained before the experiment. None of these observers participated in Experiment 1 except for RA.

\section{Stimuli and procedures}

The stimuli used and the viewing condition were largely the same as in Experiment 1. Eye movement of the observers during movement of the fixation was recorded using the EyeLink CL Tracker
(SR Research, ON, Canada) to confirm that each of the observers were tracing the fixation cross steadily with their eyes throughout the experiment. Drift correction for the position of eye fixation was carried out at the beginning of each trial. Upon initiation of a trial, the fixation cross appeared at $10.200^{\circ}$ to the left of the center of screen for $1000 \mathrm{~ms}$, and then moved horizontally to the same distance to the right of the screen center at a constant speed of $11.334^{\circ}$ /s within a 1800 -ms interval, during which the observers were required to trace at the fixation with eyes. The fixation stayed at its final position for $1000 \mathrm{~ms}$ before it disappeared. In the reference stimulus, the target circle appeared and stayed stationary at $4.533^{\circ}$ below the center of screen when the fixation stimulus arrived at $5.667^{\circ}$ to the left of the center of screen, and disappeared as the fixation arrived at the same distance to the right of the center (resulting in a presentation duration of $1000 \mathrm{~ms}$ ). The comparison stimulus was then presented, in which the fixation cross appeared and moved on the screen in the same manner as in the reference stimulus within a 1800 -ms interval. In the Moving condition, the target circle appeared (at $4.533^{\circ}$ above the vertical level of the fixation) and moved along with the fixation stimulus when the fixation stimulus arrived at $4.533 \pm 0.136^{\circ}, 5.667 \pm 0.136^{\circ}$, and $6.800^{\circ} \pm 0.136^{\circ}$ to the left of the center of screen, and disappeared as the fixation arrived at the same distance to the right of the center, for 800,1000 , and $1200 \mathrm{~ms}$ Duration conditions respectively. In the Static condition, the target circle appeared, remained stationary at $4.533^{\circ}$ above the center of screen without movement, and disappeared when the fixation stimulus arrived at the aforementioned positions for the Moving condition. After its movement, the fixation cross stayed at its final position for $1000 \mathrm{~ms}$ before returning to the center of screen, where the observer gave the response by judging which stimulus had a longer duration. Figure 3 illustrates the flow of a trial in Experiment 2.

Trials of 2 Motion conditions $\times 3$ Duration conditions $\times 3$ Starting-Ending positions were presented in pseudorandom order with 6 repetitions, resulting in a total of 108 trials. Including time for calibration of the eye tracker, the task required about 20-25 min to complete.

\section{Control experiment}

In parallel with Experiment 1, a control experiment was conducted using a moving reference stimulus to examine the possibility of novelty effect of the moving comparison stimulus. In the control experiment, the reference stimulus (1000 ms) always moved across the screen from $5.667^{\circ}$ to the left of the fixation, to the same distance to the right of the fixation instead of being stationary. Each observer also performed the main or control experiment first in counter-balanced order. A break of at least 5 min was given in between.

\section{RESULTS AND DISCUSSION}

We calculated for each observer the average moving speed of eye trace from the eye tracking data across all trials in the main experiment and confirmed that the speed were not significantly different between the Static $(M=10.171 \%, \mathrm{SD}=0.562 \% \mathrm{~s})$ and Moving $\left(M=10.278^{\circ} / \mathrm{s}, \mathrm{SD}=0.577^{\circ} / \mathrm{s}\right)$ condition of the target stimulus $[t(11)=-1.496, p=0.163]$, thus showing that the observers were tracing the fixation steadily during both conditions (actual 


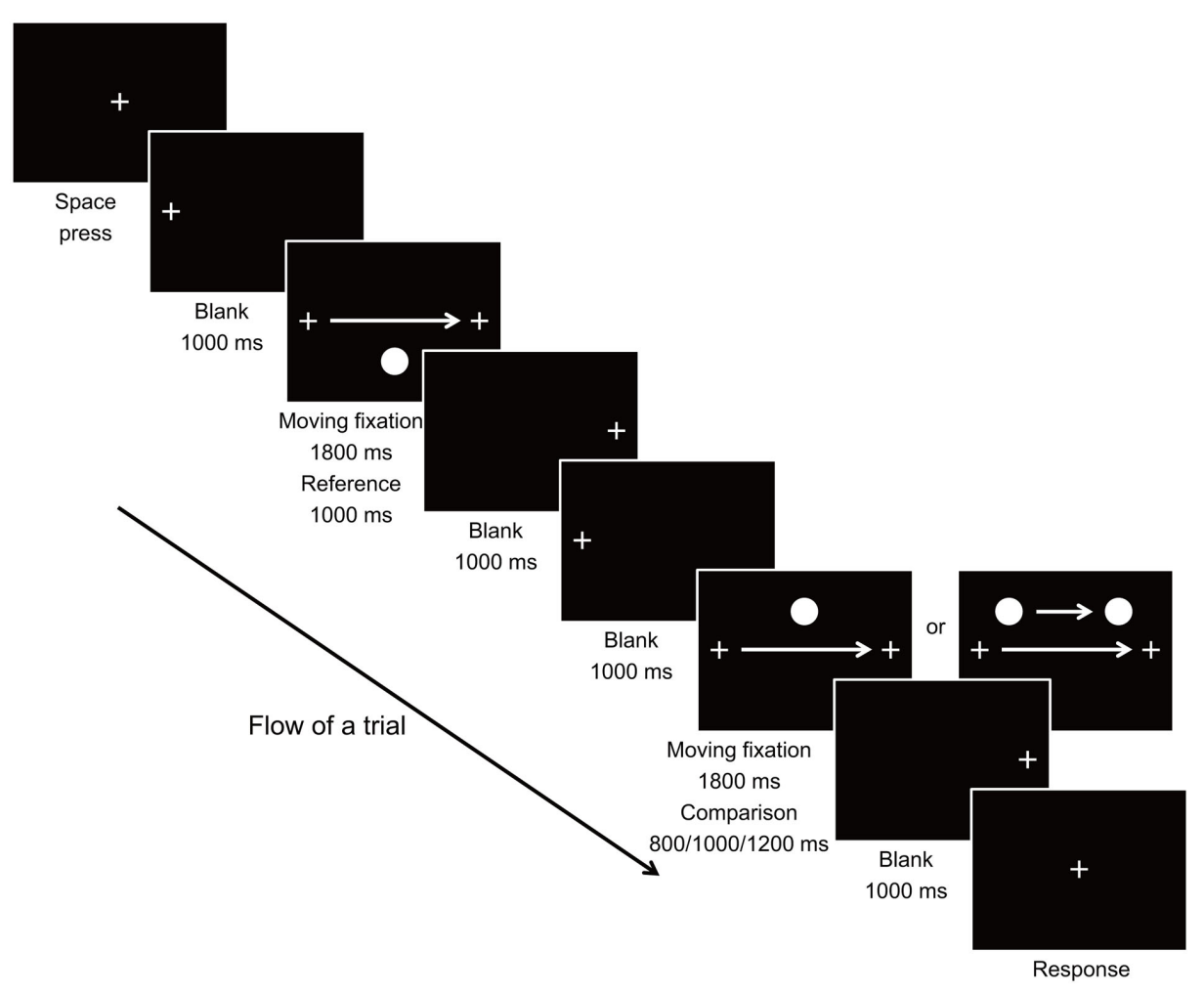

FIGURE 3 | A schematic diagram depicting the flow of a trial in Experiment 2 (main experiment).

moving speed of fixation cross $\left.=11.334^{\circ} / \mathrm{s}\right)$. No significant difference in moving speed of eye trace was found also for the control experiment [Static: $M=10.324^{\circ} / \mathrm{s}, \mathrm{SD}=0.599^{\circ} / \mathrm{s}$; Moving: $\left.M=10.326^{\circ} / \mathrm{s}, \mathrm{SD}=0.470^{\circ} / \mathrm{s} ; t(11)=-0.023, p=0.982\right]$.

For perception of time, the proportion of trials that the duration of the target stimulus was perceived to be longer than the reference stimulus was computed for each Motion condition, and is shown in Figure 4.

The same procedure of $2 \times 3$ repeated measures ANOVA with Motion (Static/Moving) and Duration (800/1000/1200 ms) as the within-subject factors as in Experiment 1 was performed on the data of the main experiment (stationary reference) to examine effect of retinal motion of object on perceived time duration (Figure 4A). Results of the ANOVA indicated that the main effect of Motion was highly significant $[F(1$, $11)=31.080, p<0.001]$, which was also true for that of Duration $[F(2,22)=56.407, p<0.001]$. But the Motion $\times$ Duration interaction was not significant $[F(2,22)=2.249, p=0.129]$. Post hoc $t$-tests reported significant differences between Static and Moving conditions at all the presentation durations of $800 \mathrm{~ms}[t(11)=-3.810, p=0.009], 1000 \mathrm{~ms}[t(11)=-5.965$, $p<0.001]$, as well as for $1200 \mathrm{~ms}[t(11)=-5.104, p=0.001$; all Bonferroni-corrected].

The $2 \times 3$ repeated measures ANOVA (Motion $\times$ Duration) was also done on the control experiment data (Figure 4B). Analysis showed significant main effects for Motion $[F(1,11)=38.498$, $p<0.001]$ and Duration $[F(2,22)=77.986, p<0.001]$, also for the Motion $\times$ Duration interaction $[F(2,22)=6.045, p=0.008]$.

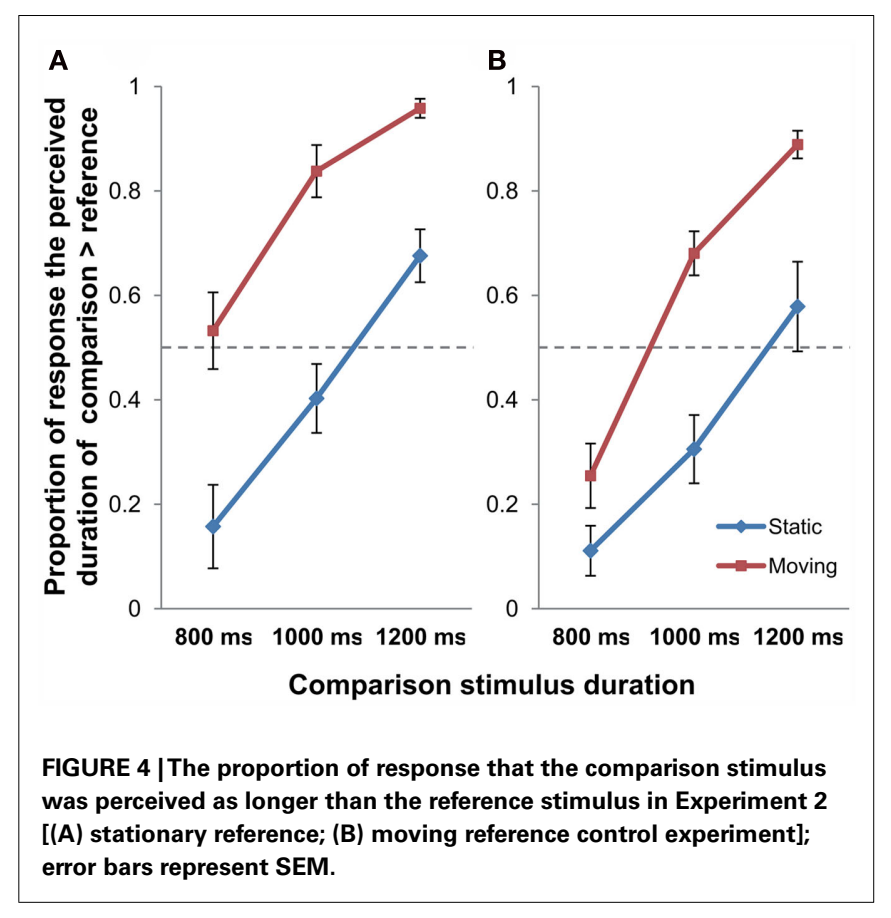

Post hoc $t$-tests revealed that the difference between Static and Moving conditions was significant for $800 \mathrm{~ms}[t(11)=-4.640$, $p=0.002], 1000 \mathrm{~ms}[t(11)=-5.348, p<0.001]$, as well as for $1200 \mathrm{~ms}[t(11)=-4.481, p=0.003]$ presentation duration 
conditions (all Bonferroni-corrected). In echo with Experiment 1 , this control experiment has again shown that the observed time dilation effect was motion-based, but not due to novelty effect of stimuli.

In sum, the findings from Experiment 2 suggested that the time dilation effect associated with object movement depends on perceived object movement represented in the brain, but not the positional shift of the image projected on the retina.

\section{GENERAL DISCUSSION}

In the present study, we have demonstrated the time dilation effect when the object physically moved in both retinotopic and objective space (Experiment 1) and when there was a physical and perceived motion but no relative motion to the retina (Experiment 2). Rather than basing on retinal displacement, the brain appeared to base on a representation which stores the real world locations and displacements of object to compute the duration of a visual event. Our result is in line with the previous suggestion regarding the existence of a spatiotopic neural mechanism responsible for linking space and time in the human brain (Burr et al., 2007), and that the perception of time of brief visual events might be processed at a stage beyond V1 (which directly receives retinal inputs) where stronger functional interactions occurs (Pariyadath and Eagleman, 2007).

In visual events, duration has to be signaled by the onset and offset at certain spatial locations, and sometimes displacement across locations (i.e., motion). Humans need to use spatial information in order to make judgments about duration of visual events, but not necessarily vice versa (Casasanto and Boroditsky, 2008). Spatial and motion processing of the visual scene therefore must be taken into account in processing the duration of the event. From results of neuroimaging studies, the parietal cortex has been shown to be important for the processing of temporal information. In a series of temporal discrimination experiments, Bueti et al. (2008) applied repetitive transcranial magnetic stimulation (rTMS) at areas including the MT/V5 (which are well known of responsible for spatial and motion processing) of human subjects to study whether disruption of brain activities in these areas would affect time perception. They found that the right posterior parietal area is important for the timing of both auditory and visual stimuli, while MT/V5 were necessary only for timing of visual events. This might imply that, instead of being processed by a specific device of pacemaker, time of visual events might be processed with the involvement of a network of different functional areas in the brain. Time has also been suggested to be encoded in high dimensional states of neural networks (Karmarkar and Buonomano, 2007). Event-related functional magnetic resonance imaging (fMRI) study has also revealed the neural substrates of the time dilation, including the anterior insula and anterior cingulate cortex that are important for cognitive control and subjective awareness, for looming and receding visual stimuli which suggest motion in depth (Wittmann et al., 2010). This supports the notion that time perception is associated with self-referential processing, that the physically stationary/moving status of objects relative to the observer is taken as a critical factor by the brain in the processing of subjective time.
Nevertheless, it remains difficult to make a firm conclusion as inconsistent findings regarding the locus of processing for time perception have been found. In the study of Johnston et al. (2006), adaptation to fast moving grating induced time compression effect for stimuli presented at the adapted area, and the effect remained even when the orientation of the adaptor and test grating differed by $90^{\circ}$, whereas the effect disappeared when the test grating was presented at a location only $1^{\circ}$ of visual angle away from the adaptation. In a later study, Bruno et al. (2010) reported that as the adaptor continuously moved across head-centered coordinates while maintaining at a fixed retinal position, duration compression effect remained. Strong time compression effect was found under retinotopic adaptation, but no significant change in perceived duration under spatiotopic adaptation. Based on these results, the authors argued for an early locus of the adaptation-based time compression effect.

But it might be possible that the mechanism underlying time perception with visual adaptation is different from that for visual motion. The classic change model as described by Poynter and Homa (1983) maintains that the amount of change occurring during a time interval determines the perception of time. A stimulus with more changes lengthens the subjective experience of time. In comparison with stationary stimulus, a moving stimulus carries continuous change in location (and more change for faster moving stimuli), resulting in a longer duration as interpreted by the brain (Brown, 1995). Some scholars have suggested the possibility that attention and the amount of perceptual information to be processed determine the perception of time (Hemmes et al., 2004; Tse et al., 2004). Presenting to observers a series of stimuli, Tse et al. (2004) demonstrated that a low probability oddball stimulus could create a subjective perception of longer duration than high probability stimulus, though their objective durations were same. With the duration reproduction method, Brown and Boltz (2002) found that an increased mental workload and poor event structure could lead to inaccuracy to the reported duration of melodies and prose passages, demonstrating the relevance of attention in time estimation. Predictability (Ulrich et al., 2006; Pariyadath and Eagleman, 2007), exogenous visual attention (Seifried and Ulrich, 2011), and onset time of stimulus (Rose and Summers, 1995; Kanai and Watanabe, 2006) could also play a role in lengthening the perceived duration of events. Pariyadath and Eagleman (2007) proposed that, the amount of neural energy needed by the brain to represent a stimulus relates to the coding efficiency (Eagleman and Pariyadath, 2009), and correlates with the perceived duration. A suppressed response (as in the situation of repeated stimulus) therefore leads to perception of a shorter duration. Linking the ideas to time perception during visual motion, a moving stimulus at a high speed would carry more perceptual information than stationary ones, and require higher level of attention and neural energy to keep track of, leading to lengthened time perceived. A stationary stimulus, on the other hand, may result in suppression of neural response, resulting in shorter perceived time comparing to moving ones.

To conclude, the present study replicated the time dilation effect with eye fixation, and further demonstrated that the time dilation effect of object motion relies on the spatiotopic representation 
about physical positions of objects in the world, but not retinotopic positions. These results are in support with the idea that the perception of time for brief visual events depends on the interactions among extended brain areas occurring at a higher level of processing beyond the primary visual cortex.

\section{REFERENCES}

Aschersleben, G., and Müsseler, J. (1999). Dissociations in the timing of stationary and moving stimuli. $J$. Exp. Psychol. Hum. Percept. Perform. 25, 1709-1720.

Brainard, D. H. (1997). The Psychophysics Toolbox. Spat. Vis. 10, 433-436.

Brown, J. F. (1931). On time perception in visual movement fields. Psychol. Res. 14, 233-248.

Brown, S. W. (1995). Time, change, and motion: the effects of stimulus movement on temporal perception. Percept. Psychophys. 57, 105-116.

Brown, S. W., and Boltz, M. G. (2002). Attentional processes in time perception: effects of mental workload and event structure. J. Exp. Psychol. Hum. Percept. Perform. 28, 600-615.

Bruno, A., Ayhan, I., and Johnston, A. (2010). Retinotopic adaptationbased visual duration compression. J. Vis. 10, 1-18.

Bueti, D., Bahrami, B., and Walsh, V. (2008). Sensory and association cortex in time perception. J. Cogn. Neurosci. 20, 1054-1062.

Burr, D., Tozzi, A., and Morrone, M. C. (2007). Neural mechanisms for timing visual events are spatially selective in real-world coordinates. Nat. Neurosci. 10, 423-425.

Casasanto, D., and Boroditsky, L. (2008). Time in the mind: using space to think about time. Cognition 106, 579-593.

Eagleman, D., and Pariyadath, V. (2009). Is subjective duration a signature of coding efficiency? Philos. Trans. R. Soc. Lond. B Biol. Sci. 364, 1841-1851.

Fraisse, P. (1984). Perception and estimation of time. Annu. Rev. Psychol. $35,1-36$.

Hemmes, N. S., Brown, B. L., and Kladopoulos, C. N. (2004). Time perception with and without a concurrent nontemporal task. Percept. Psychophys. 66, 328-341.

Johnston, A., Arnold, D. H., and Nishida, S. (2006). Spatially localized distortions of event time. Curr. Biol. 16, 472-479.

Kanai, R., Paffen, C. L., Hogendoorn, H., and Verstraten, F. A. (2006). Time dilation in dynamic visual display. $J$. Vis. 6, 1421-1430.

Kanai, R., and Watanabe, M. (2006). Visual onset expands subjective time. Percept. Psychophys. 68, 1113-1123.

Kaneko, S., and Murakami, I. (2009). Perceived duration of visual motion increases with speed. J. Vis. 9, 1-12.

Karmarkar, U. R., and Buonomano, D. V. (2007). Timing in the absence of clocks: encoding time in neural network states. Neuron 53, 427-438.

Matthews, W. J. (2011). How do changes in speed affect the perception of duration? J. Exp. Psychol. Hum. Percept. Perform. 37, 1617-1627.

Matthews, W. J., Stewart, N., and Wearden, J. H. (2011). Stimulus intensity and the perception of duration. J. Exp. Psychol. Hum. Percept. Perform. 37, 303-313.

Morrone, M. C., Ross, J., and Burr, D. (2005). Saccadic eye movements cause compression of time as well as space. Nat. Neurosci. 8, 950-954. The subjective size of visual stimuli affects the perceived duration of their presentation. Percept. Psychophys. 69, 952-957.

Ono, F., and Kitazawa, S. (2009). The effect of marker size on the perception of an empty interval. Psychon. Bull. Rev. 16, 182-189.

Ono, F., and Kitazawa, S. (2010). The effect of perceived motion-in-depth on time perception. Cognition 115, 140-146.
Ono, F., and Kawahara, J. (2007).

\section{ACKNOWLEDGMENTS}

This work was supported by grants from Japan Society for the Promotion of Science to Ricky K. C. Au, Fuminori Ono, Katsumi Watanabe and Japan Science and Technology Agency to Katsumi Watanabe.

Pariyadath, V., and Eagleman, D. M. (2007). The effect of predictability on subjective duration. PLOS ONE 2, e1264. doi:10.1371/journal.pone.0001264

Pelli, D. G. (1997). The VideoToolbox software for visual psychophysics: transforming numbers into movies. Spat. Vis. 10, 437-442.

Poynter, W. D., and Homa, D. (1983). Duration judgment and the experience of change. Percept. Psychophys. 33, 548-560.

Rachlin, H. C. (1966). Scaling subjective velocity, distance, and duration. Percept. Psychophys. 1, 77-82.

Roelofs, C. O. Z., and Zeeman, W. P. C. (1951). Influence of different sequences of optical stimuli on the estimation of duration of a given interval of time. Acta Psychol. 8, 89-128.

Rose, D., and Summers, J. (1995). Duration illusions in a train of visual stimuli. Perception 24, 1177-1187.

Schiffman, H. R., and Bobko, D. J. (1974). Effects of stimulus complexity on the perception of brief temporal intervals. J. Exp. Psychol. 103, 156-159.

Schütz, A. C., and Morrone, M. C. (2010). Compression of time during smooth pursuit eye movements. Vis. Res. 50, 2702-2713.

Seifried, T., and Ulrich, R. (2011). Exogenous visual attention prolongs perceived duration. Atten. Percept. Psychophys. 73, 68-85.

Terao, M., Watanabe, J., Yagi, A. and Nishida, S. (2008). Reduction of stimulus visibility compresses apparent time intervals. Nat. Neurosci. 11, 541-542.

Tse, P. U., Intriligator, J., Rivest, J., and Cavanagh, P. (2004). Attention and subjective expansion of time. Percept. Psychophys. 66, 1171-1189.

Ulrich, R., Nitschke, J., and Rammsayer, T. (2006). Perceived duration of expected and unexpected stimuli. Psychol. Res. 70, 77-87.

Verstraten, F. A. J., Kanai, R., Hogendoorn, H., and Paffen, C. L. E. (2005). Visual motion expands perceived time. Perception 34(Suppl.), 30.

Wenke, D., and Haggard, P. (2009). How voluntary actions modulate time perception. Exp. Brain Res. 196, 311-318.

Wittmann, M., van Wassenhove, V., Craig, A. D., and Paulus, M. P. (2010). The neural substrates of subjective time dilation. Front. Hum. Neurosci. 4:2. doi:10.3389/neuro.09.002.2010

Xuan, B., Zhang, D., He, S., and Chen, X. (2007). Larger stimuli are judged to last longer. J. Vis. 7, $1-5$.

Conflict of Interest Statement: The authors declare that the research was conducted in the absence of any commercial or financial relationships that could be construed as a potential conflict of interest.

Received: 29 November 2011; paper pending published: 25 December 2011; accepted: 13 February 2012; published online: 28 February 2012.

Citation: Au RKC, Ono F and Watanabe $K$ (2012) Time dilation induced by object motion is based on spatiotopic but not retinotopic positions. Front. Psychology 3:58. doi: 10.3389/fpsyg.2012.00058

This article was submitted to Frontiers in Perception Science, a specialty of Frontiers in Psychology.

Copyright (c) $2012 \mathrm{Au}$, Ono and Watanabe. This is an open-access article distributed under the terms of the Creative Commons Attribution Non Commercial License, which permits noncommercial use, distribution, and reproduction in other forums, provided the original authors and source are credited. 